\title{
A Software Interface as a Teaching Tool for Analysing Conformal Antenna Arrays
}

\author{
J. P. González-Coma, R. Maneiro-Catoira, P. M. Castro, J. Brégains
}

*Department of Electronics and Systems, Universidade da Coruña (Spain)

\begin{abstract}
Electromagnetic laboratory teaching activities greatly benefit from utilizing Information and Communication Technologies (ICTs), due to the fact that the understanding of concepts that are difficult to be communicated using traditional approaches becomes easier. We have developed a graphical interface, designed with MATLAB ${ }^{\circledR}$, that represents a very useful tool to convey concepts related to configuration and radiation of conformal antennas to students that are not experts in that particular field.
\end{abstract}

Keywords: Conformal antenna arrays, ICTs, MATLAB, software interface, teaching tool.

\section{Introduction}

Previous researches have shown that students learn and retain much more when they directly experiment in the laboratory, as opposed to when they only listen or see concepts in traditional classroom lectures, as explained in works by Shyr (2010), Bonwell (1991) and McKeachie (1994). Learning is a dynamic activity, and the process of comprehension and understanding of a set of interrelated concepts is greatly enhanced if the student takes an active part in such an assimilation process. In the case of proactive students, the aforementioned dynamism practically does not depend on their teachers, as they march at almost self-taught pace. But for less determined students, any external supplementary tool can help to fill the motivational gaps. It is clear that the number and nature of the variables that modify motivation conform a complex topic that deserves a separate research -see for example Christensen (2010), Hanrahan (1998) and Henning (2007)-, but no doubt the nearer the learning tools to amusing activities, the higher the enthusiasm that will catalyse the learning process towards successful outcomes. The tremendous ubiquity of computer systems nowadays allows teachers to reach this purpose by focusing the design of learning activities on software tools that result to be entertaining for the students, in some cases up to the limit of creating videogames, Mitchell (2004). Under these views it is of no surprise that the European Higher Education Area -EHEA, see Declaración de Soborna (1998) and Declaración de Bolonia (1999)- promotes, among other things, the utilization of Information and Communication Technologies (ICTs) in order to reinforce the self-based learning of students. As nowadays students are totally familiarized with ICTs, it is easy for them to learn the use of new software tools without complex memorization requirements; this feature, together with the fact that most of them play or have played videogames on a regular basis, deepens the justification of professors to design new computer applications that facilitate the learning process. Moreover, as the combination of visual with conceptual learnings is much more effective than conceptual learning alone -widely employed by traditional methodologies-, graphic-based teaching tools are particularly useful for handling those concepts that sometimes are hidden by abstract ideas, or even in cases where the ideas are simple, but the mathematical language used to describe them is so entangled that such ideas easily become obscured. This latter is the case of, for example, antenna theory, whose conceptual modelling has easy-to-follow concepts, but whose mathematics require from the students additional efforts, compensated by necessary, and sometimes tiresome, training sessions. 
In agreement with the abovementioned perspectives, we have designed a teaching tool that makes use of ICTs and that allows the teacher to facilitate the students' understanding of the subject of antenna radiation patterns, successfully included in the syllabus of the Master's course program "Information Technologies and Communications in Mobile Networks (ITCRM)", and in the subject "Mobile and Wireless Networks (RM)" corresponding to the fourth year of the Degree in Computer Science at the University of A Coruña (Spain). The tool, described in this paper, consists of a novel graphical interface, designed with MATLAB ${ }^{\circledR}$, and intended to be used to convey concepts related to antenna radiation. Such an interface is capable of representing both the spatial and electrical configurations of a conformal antenna array, as well as the different patterns radiated by it. Thanks to this computerassisted learning, students are able to perform, at will, instructive experiments to further understand the theoretical concepts given previously in the traditional classroom lectures. The utility of the tool is illustrated with a sample lesson.

\section{Material and Methods}

An arrangement of single electromagnetic radiating elements is called an antenna array. According to their spatial distribution, they are called linear -when the elements are aligned along a straight line, planar -when arranged on a plane, or conformal -if distributed on a specific three-dimensional surface. Antenna arrays are designed in order to radiate electromagnetic energy towards desired spatial locations, focusing on desired regions. Another important feature of antenna arrays is related to their electromagnetic feeding distribution, which, basically, represents the amount of energy used to supply each element. Each element of an array has its own radiation diagram, and also a feature called polarization, which represents the spatial direction of the oscillation of the radiated field. The proper combination of both the spatial and feeding configurations, taking also into account the polarization arrangement, allows the designer to obtain the required radiated energy spatial distribution.

Conformal antennas are especially difficult to characterize, since the polarizations of their elements are usually not aligned among themselves, a feature that considerably increases the design complexity. In spite of the fact that they nowadays constitute a well-studied subject, as seen in Josefsson (2006), surprisingly it is not easy to find any freely-distributed software tool capable of representing visual information about the spatial distributions of both their physical configuration and their electromagnetic radiation. The currently available tools correspond to either commercial electromagnetic simulators that make use of well-known numerical techniques, such as Finite Difference Time Domain (FDTD), Method of Moments (MM), etc., see Davidson (2010), or a recent MATLAB ${ }^{\circledR}$ toolbox called "Phased Array System Toolbox" -The Matlab Group (2011)-, neither of them freely available. Normally, those tools are expensive, and, which is also important, their handling entails considerable technical knowledge from the users. The latter drawback is remarkably important when the tools are required to be used with learning purposes, since students usually have a limited amount of time to acquire the necessary, and complex, handling skills.

The designed software tool described here deals efficiently with the abovementioned problems: it requires little previous knowledge from the user and its source code can be freely obtained, provided he/she has MATLAB at his/her disposal -as happens with the students of our courses. Such a free tool developed by the authors is also relevant in the sense of strengthening the homework of students without the need of being 
present in a traditional lecture, thus ensuring two basic principles of the EHEA: firstly, by promoting the Student-Centred Learning (SCL), since it increases the active learning with a major motivation in the students, Alonso (1998) and Colas (2009), and secondly, by exploiting a new relationship between them and the teacher, given that the traditional way of teaching and learning is substituted or complemented by the employment of ICTs that simulate the real environment. Thus, they can empirically test different configurations with several parameters that would not be approached using the traditional oral questions addressed to the professor for solving doubts. This tool is also intended to reinforce the interaction teacher-student, as represented in Figure 1, and it is exclusively oriented to university studies due to the high technical knowledge required to the students when they are bound to justify the presented results, which also contributes to promote their criticism, in order to avoid automatic behaviours that most of the time obstructs the reasoning process.

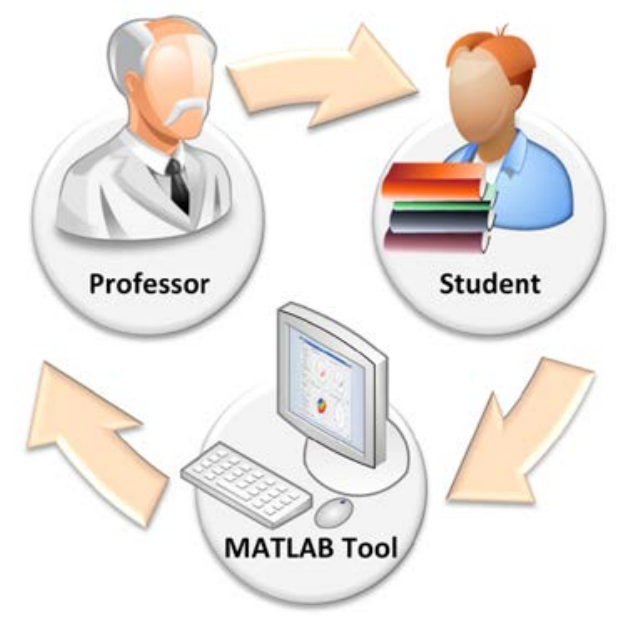

Figure 1. Interaction between student and professor using the teaching tool presented in this work. Source of the icons: www.iconarchive.com

\section{Description of the Tool: Example and Discussion}

During the course, the students are given, through regular lectures, the mathematical description of the physical models that represent conformal array antennas. Part of the mathematical description related with the presented tool can be found in the papers by Brégains et al. (2004, 2010, and 2011).

We proceed now to briefly describe the proposed tool, including an example.

By typing the sentence (The MATLAB Group, 2008) >> guide ConfPolPow3D.fig in the MATLAB ${ }^{\circledR}$ command line, the MATLAB GUI compiler will be opened. In this case, the designed ConfPolPow3D program will not run immediately, and just its interface will appear on the main window. In this manner, the user is able to edit both the outer aspect of the interface (add buttons, text boxes, etcetera), and the source code that will follow the commands actions that such an interface will receive from the user.

After having finished its edition, the program will be executed by using the Ctrl+T shortcut, for example. Afterwards its main interface will appear, see Figure 2. As it can be seen, there are separate frames corresponding to: Array Configuration (for both the spatial and the electrical configuration of the array), Pattern Options (2D and 3D features for the field spatial distribution) and Visualization Space (four sub-frames within which there will appear, on the one hand, the visual representation of the array 
spatial and electrical distribution, and, on the other hand, the plots corresponding to the field radiated by it).

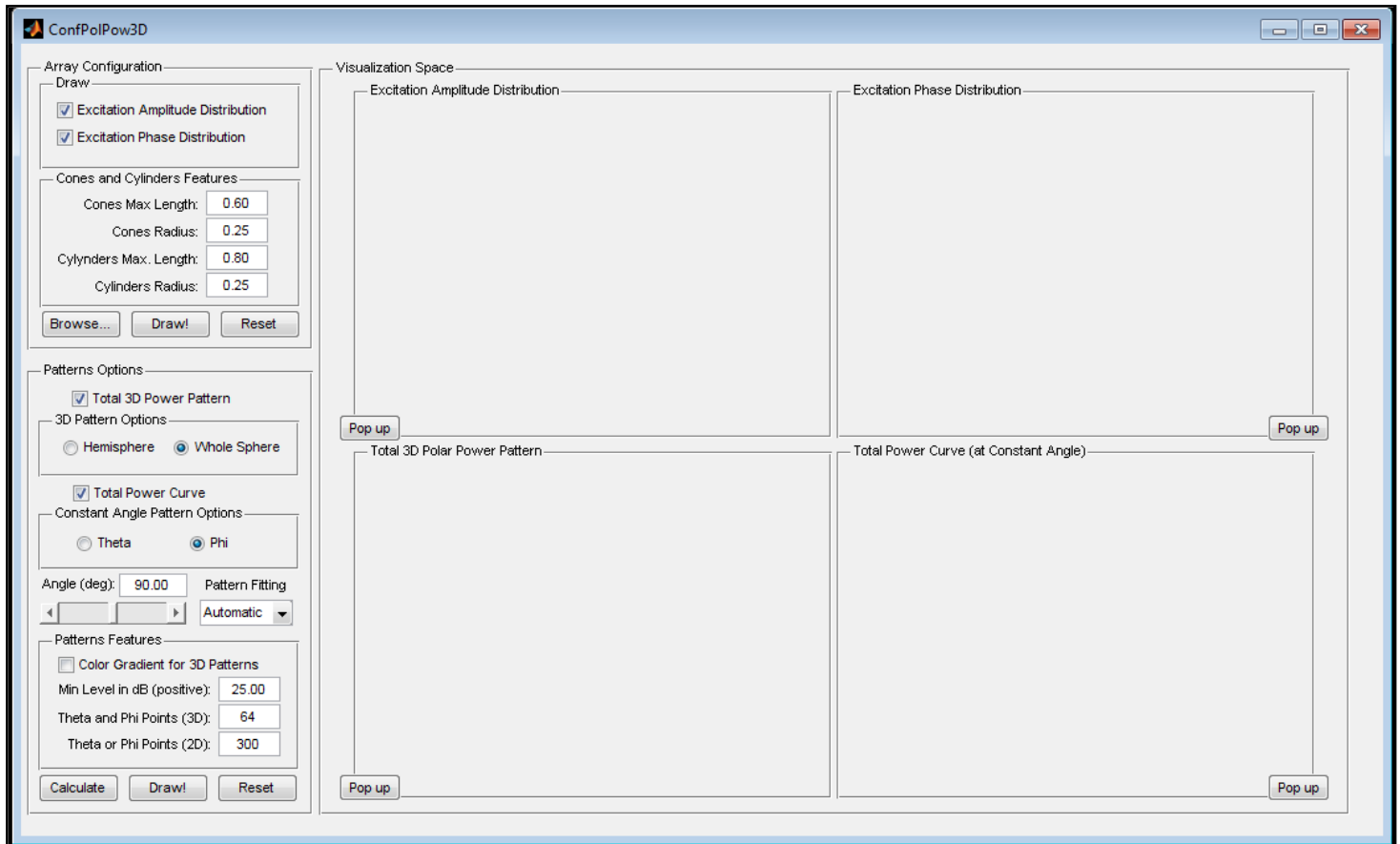

Figure 2. Main interface of the described tool.

When the student clicks on the "Browse..." button from the Array Configuration frame, see Figure 2, the program proceeds to load into memory the conformal array configuration. This is done by reading a selected text file (.dat extension). The data corresponding to each radiating element must have been previously introduced by the student into such a text file. After having selected the configuration file, the program will load the data and run some previous calculations, informing, by means of a window message if there have been any errors when accessing the file. If the data have been correctly loaded, then both the number and type of elements (patches, dipoles, or isotropic elements, see Balanis, 2005, and Brégains, 2003 and 2005) will be shown to the student, indicating also that both the electrical and spatial configurations of the array are ready to be visualized (see Figure 3). Figure 4 shows an example when an array composed of eleven dipoles arranged on a cylinder has been successfully loaded.

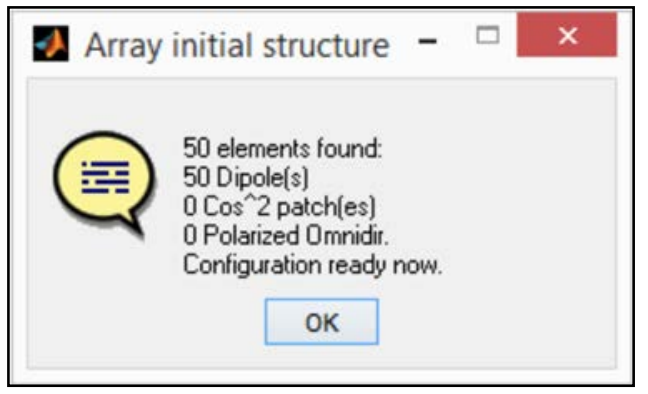

Figure 3. Message box indicating that the program successfully loaded an array configuration composed of fifty dipoles (Brégains, 2003 and 2005).

Before proceeding to plot such configurations, the student will be able to establish the relative size, given in $\lambda$ units, of the maximum amplitude and phases (feeding of the elements), represented by oriented cones and non-oriented cylinders, respectively. The orientation of each cone represents the $z_{n}$ direction of the local coordinate system of 
each element (Brégains et al., 2011). The heights and radius of those geometric figures representing amplitudes and phases can be configured by the controls gathered on panel Cones and Cylinders Features, see Figure 3.

Once the configuration is loaded, by clicking on Draw! button from the Array Configuration frame, the program will proceed to plot the array relative amplitude and/or phase distributions, according to the selections performed within the Draw panel (at least one of the Excitation Amplitude Distribution and Excitation Phase Distribution checkboxes must be selected, see Figure 2). The corresponding plots will appear on the upper half of the Visualization Space, see Figure 4. The student will have then at his/her disposal the option of erasing the graphics by clicking on Reset button in the Array Configuration panel, or of obtaining detached figures, capable of being adequately edited, by clicking on any of the Popup buttons, located on the bottom vertex of the given plots on the Excitation Amplitude Distribution or Excitation Phase Distribution frames, see Figure 2.

Once the array configuration has been loaded, it will be necessary to set the power pattern parameters to be further calculated and visualized: on the one hand, it will be necessary to indicate if the 3D pattern is requested to be calculated (Total 3D Power Pattern at the top of the Patterns Options panel, see Figure 2), over the whole space (Whole Space) or the southern hemisphere (Hemisphere), and, on the other hand, the student will have to specify a 2D pattern by setting either $\theta$ =constant or $\varphi=$ constant (which are the usual spherical coordinates), at an angle specified by the Angle (deg) edit text box, this latter having the option of being selected with the help of a slider located below it. In Figure 3, for example, within the Constant Pattern Options panel, there has been selected the Phi radio button option, considering Angle (deg): 90.00. This means that the power radiated pattern is calculated by taking $\varphi=90^{\circ}$, with $\theta \in(0$, $\left.180^{\circ}\right)$.

The Pattern Fitting options list will set the type of limit adjustment of the axis box containing the 3D pattern. The student will be able to select the more adequate solution according to his/her: Automatic, corresponding to the axis auto MATLAB ${ }^{\circledR}$ option; Packed, setting axis fill, or Level, establishing the box limits (i.e., the limits of the box enclosing the coordinate axes of the visualized power patterns) between $-l e v_{\min }$ and $l e v_{\min }$, being $l e v_{\min }$ the value indicated on Min Level in $d B$ (Positive) text box, from the Patterns Features panel.

The Theta and Phi Points (3D) text will indicate the program the number of points selected for sweeping the $\theta$ and $\varphi$ angles on the 3D plot: in other words, the number of $\varphi$ points taken from 0 to $360^{\circ}$, and the number of $\theta$ points taken from 0 to $180^{\circ}$ (in case of whole space) or from 0 to $90^{\circ}$ (in case of hemisphere) on which the radiated power pattern is calculated to be finally represented. The same is applicable for the Theta or Phi Points (2D) edit text, that indicates the number of points for sweeping the $\varphi$ (for $\theta=$ constant ) or the $\theta$ (for $\varphi=$ constant) angles on the planar patterns.

The Color Gradient for 3D Patterns option will set a colour map on the 3D power surfaces, with corresponding colour bars that will inform the student about the concerned levels (unless the field is null, in which case those bars will be omitted). If such an option is not selected, then the 3D patterns will be drawn with uniform colours and no level bar. In both cases, the axis box will be given with ticks and labels on the $x, y$, and $z$ axes. 
When clicking the Calculate button, the program will perform the computations of all the fields (total, and a few components, Brégains et al. 2011) specified by the student (3D and/or 2D patterns). Figure 4 shows the 3D patterns obtained for the array configuration presented above, after the corresponding Calculate and Draw! buttons have been clicked.

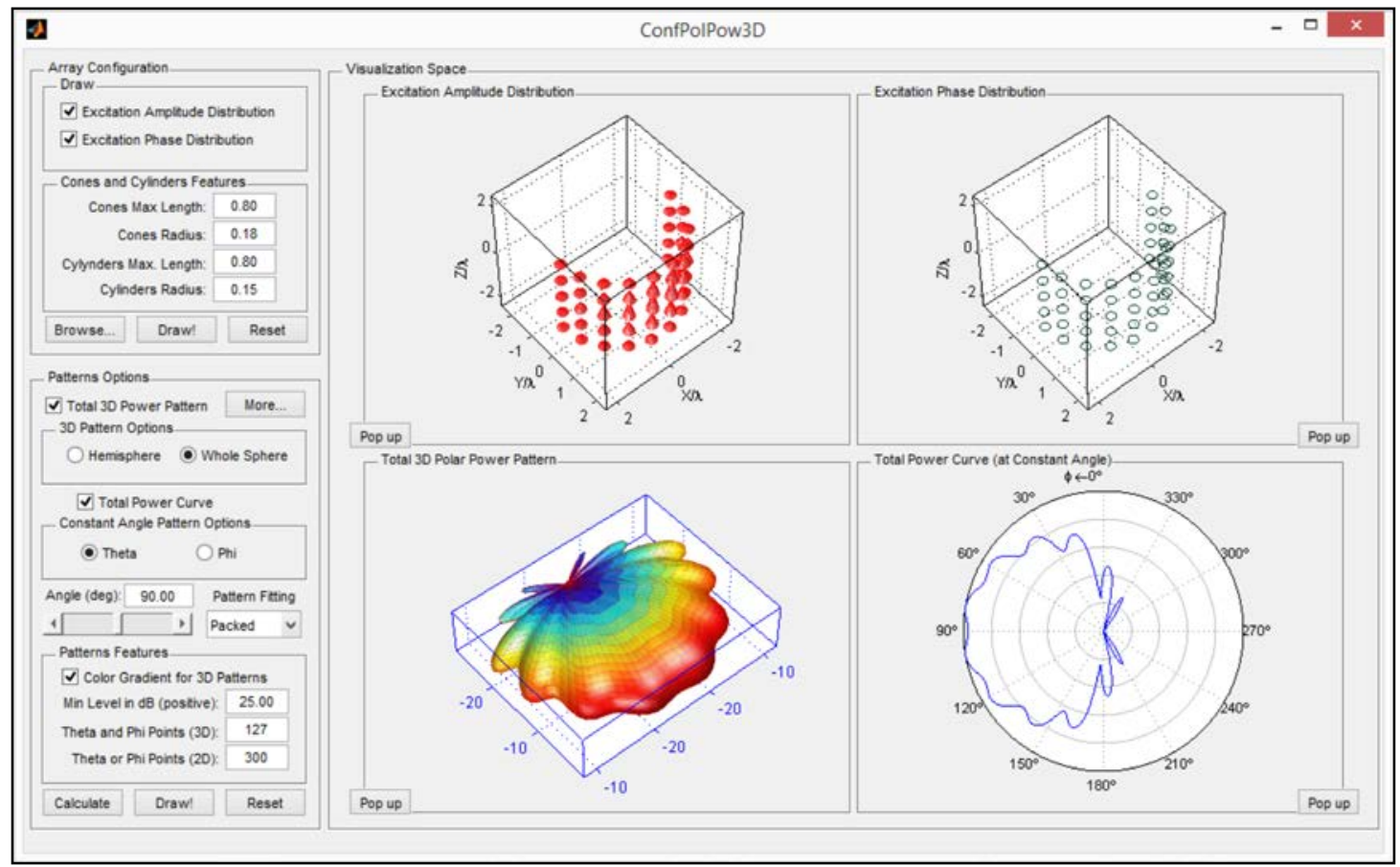

Figure 4. Outward appearance of the main interface of the tool once the spatial and electrical configuration of the array, as well as its radiated patterns, have been plotted. Plots correspond to a semicylindrical array of 50 dipoles (Brégains, 2003 and 2005) backed by a ground plane. Top: electrical and spatial array configuration. Bottom: Total power (3D and 2D) patterns.

The source code is performed so as to give the students the possibility of having at their disposal the use of several options of the array elements. In the first attempt of our code we have considered three options: a half-wavelength dipole, a circular patch and finally an (unrealistic, but useful for further testing the program) omnidirectional element.

With the "Popup" button of the 3D diagram the program will plot, in separate windows, the graphics corresponding to three different components of the field. There is also a corresponding Pop up button for 2D plots, see Total Power Curve (at Constant Angle) panel in Figure 4. In all these cases, including the plot belonging to such a panel, we have used a function that performs planar polar plots.

\section{Conclusions}

This tool has been used as a test in the lectures corresponding to the Master's course program "Information Technologies and Communications in Mobile Networks (ITCRM)" during the academic year 2011/12 and in the lectures of "Mobile and Wireless Networks (RM)" introduced in 2013/2014 according to the new studies plan designed in the context of EHEA up to now. This optional subject corresponds to the fourth year of the Degree in Computer Science at the University of A Coruña (Spain). We have used this tool in conjunction with standard graphical tools in the area of antenna radiation and signal propagation, although as a further line work we are 
currently seeking the development of a unique and integrated software, thus avoiding the utilization of multiple software (neither always free nor easily available for the students). The tool was welcomed by the students, and although we did not gather yet the required information to quantitatively evaluate the impact of the use of this program on their performance, we have found a significant improvement on the quality of the management of the concepts they have to acquire since the tool was introduced.

For the visual representation of the fields generated by conformal arrays composed of elements whose patterns are well studied, and with configurations where the mutual coupling can be neglected, the presented tool constitutes a reliable alternative to the commercial programs based on known numerical techniques. The students have also the option of modifying at will the source code, which remarkably increases the utility of the tool.

In spite of the completeness of the tool, some improvements could be suggested. The possibility of writing an output file containing any of the calculated patterns is a possibly useful feature. The three components of the field for the constant $\theta$ (or $\varphi$ ) polar cuts could be represented within a single figure. Sometimes would be preferable to draw Cartesian planar plots instead of polar ones. Additional pattern attributes could be controlled by the inclusion of further controls (buttons, check boxes, etcetera), but at the price of needing more space to locate them.

Finally, it is important to remark that several results obtained with the proposed tool were successfully validated -taking into account that the negligibility of the mutual coupling between elements would cause apparent differences with respect to the more realistic results- with a commercial software tool (based on the FDTD technique). In this sense, it would be also possible, in a future work, to compare the performance of the tool introduced here with that of MATLAB -Phased Array System Toolbox, mentioned previously (MATLAB, 2011)-, something that was not performed here because our research group does not hold the corresponding tool license yet.

The source code is freely available on request to one the authors of this work (julio.bregains@udc.es). The zipped file containing the code also includes several antenna configurations, such as the one given here as example, together with a brief tutorial that would help the interested user to quickly grasp the program handling.

\section{Acknowledgements}

The authors thanks Xunta de Galicia and Ministerio de Economía y Competitividad of Spain for its financial support under grants number 2012/287 and TEC2013-47141C4-1-R.

\section{References}

Alonso Tapia, J. (1998) ¿Qué podemos hacer los profesores universitarios para mejorar el interés y el esfuerzo de nuestros alumnos por aprender? Ministerio de Educación y Cultura (Ed), Madrid: Premios Nacionales de Investigación Educativa, 151-187.

Bonwell, C.C. \& Eison, J.A. (1991). Active learning: Creating excitement in the classroom, Washington C. C. George Washington University.

Balanis, C.A. (2005). Antenna Theory. Analysis and design, 3r edition, New Jersey: Wiley-Interscience 
Brégains, J. C., Ares, F. \& Moreno, E. (2004). Visualizing the 3D Polar Power Patterns and Excitations of Planar Arrays with MATLAB, IEEE Antennas and Propagation Magazine, 46, 2004, 108-112.

Brégains, J.C. \& Ares. F. (2003). Variation in Bandwidths of Pattern Quality Parameters and Maximum Embedded Impedance Among the Solutions to Shaped Beam Synthesis Problems for Collinear Dipole Arrays, IEEE Antennas and Wireless Propagation Letters, 2, 267-268.

Brégains, J.C. \& Ares, F. (2005). Variation in Bandwidths among Solutions to Shaped Beam Synthesis Problems Concerning Linear Arrays of Parallel Dipoles, IEEE Transactions on Antennas and Propagation, 53, 563-564.

Brégains, J.C. García-Naya, J.A., Dapena, A \& González-López, M. (2010) A MATLAB Tool for Visualizing The 3D Polar Power Patterns and Excitations of Conformal Arrays, IEEE Antennas and Propagation Magazine, 52, 127-133.

Brégains, J.C., García-Naya, J.A., González-López, M. A., Castedo, L. (2011). A MATLAB Interface for Analyzing Conformal Arrays Composed of Polarized Heterogeneous Elements, IEEE Antennas and Propagation Magazine, 53, 136144.

Christensen, C.M., Horn, M.B. \& Johnson, C.W. (2010). Rethinking student motivation: Why understanding the 'job' is crucial for improving education, Boston: Innosight Institute.

Colas, P. \& De Pablos, J. (2009). La universidad en la Unión Europea. El Espacio Europeo de Educación Superior y su impacto en la docencia. Archidona: Aljibe.

Davidson, D.B. (2010). Computational Electromagnetics for RF and Microwave Engineering (2nd edition), New York: Cambridge University Press.

Declaración De Bolonia. (1999). Online: http://www.universia.es/contenidos/universidades/documentos/Universidades_do cum_bolonia.htm, Paris.

Declaración De Sorbona. (1998). Online: http://www.universia.es/contenidos/universidades/documentos/Universidades_do cum_sorbona.htm, Paris.

Hanrahan, M. (1998). The Effect of Learning Environment Factors on Students' Motivation and Learning, International Journal of Science Education, 20, 737753.

Henning, M. (2007). Students' Motivation to Learn, Academic Achievement, and Academic Advising (PhD Thesis), New Zealand: AUT University.

Josefsson, L. \& Persson, P. (2006). Conformal Array Antenna Theory and Design, New York: IEEE Press/John Wiley.

Mckeachie, W.J. (1994). Teaching Tips: Strategies, Research, and Theory for College and University Teachers, 9th ed. Lexington, MA: Cengage Learning.

Mitchell, A. \& Savill-Smith, C. (2004).The Use of Computer and Video Games for Learning, London: Learning and Skills Development Agency.

Shyr, J. W. (2010). Integrating laboratory activity into a junior high school classroom, IEEE Transactions on Education, 53, 32-37.

The Matlab Group, Inc. (2008). Matlab Function Reference: Volumes 1, 2, 3, (printed version of the corresponding MATLAB Help menu).

The Matlab Group, Inc. (2011). Online: www.mathworks.com/products/phased-array. 